\title{
Li-Yorke chaotic eigen set of the backward shift operator on $\ell^{2}(\mathbb{N})$
}

https://doi.org/10.1515/conop-2020-0105

Received February 19, 2020; accepted September 15, 2020

Abstract: In this paper, we prove our main result that the Li-Yorke chaotic eigen set of a positive integer multiple of the backward shift operator on $\ell^{2}(\mathbb{N})$ is a disk in the complex plane $\mathbb{C}$ and the union of such Li-Yorke chaotic eigen set's is the whole complex plane $\mathbb{C}$.

Keywords: Li-Yorke chaos, eigen set, backward shift, irregular vectors.

\section{Introduction}

The concept of Li-Yorke chaoticity of a continuous mapping in a topological dynamical system and the concept of irregular vectors of Hilbert space operators are well known in Chaos theory and Operator theory respectively (see [1], [2], [3], [6], [11], [13], [14]). Here, we made an attempt to link these two concepts together and introduce the Li-Yoke chaoticity of an operator in a separable complex Hilbert space. Also we defined the Li-Yorke chaotic eigen set of an operator and use this idea to prove our main result.

In this paper, we denote the unit circle as $\mathbb{T}=\{z \in \mathbb{C} /|z|=1\}$ and the unit disk as $\mathbb{D}=\{z \in \mathbb{C} /|z|<1\}$. Also we take $X$ a separable complex Hilbert space and $B(X)$ denotes the space of all bounded linear operators on $X$.

Definition 1.1. Let $T \in B(X)$. An irregular vector for an operator $T$ is an $x \in X$ such that $\lim _{\sup }\left\|T^{n} x\right\|=\infty$ and $\liminf _{n}\left\|T^{n} x\right\|=0$. The operator $T \in B(X)$ is said to be Li-Yorke chaotic if and only if there exists an irregular vector for $T$. For $T \in B(X)$, the Li-Yorke chaotic eigen set of $T$, denoted by $L Y(T)$, is defined by $L Y(T)=\{\lambda \in \mathbb{C} / T-\lambda I$ is Li-Yorke chaotic $\}$.

Definition 1.2. ([4]) For $\Omega$ a connected open subset of $\mathbb{C}$ and $n$ a positive integer, let $B_{n}(\Omega)$ denote the operators $T$ in $B(X)$ which satisfy:
(a) $\Omega \subseteq \sigma(T)=\{\omega \in \mathbb{C} / T-\omega$ not invertible $\}$
(b) $\operatorname{Ran}(\mathrm{T}-\omega)=X$ for $\omega$ in $\Omega$
(c) $\bigvee \operatorname{Ker}_{\omega \in \Omega}(T-\omega)=X$
(d) $\operatorname{dim} \operatorname{Ker}(T-\omega)=n$ for $\omega$ in $\Omega$

Lemma 1.3. ([4]) Let $T \in B_{n}(\Omega)$. If $\Omega \cap \mathbb{T} \neq \phi$, then $T$ is Li-Yorke chaotic.

We now state our main result as:

Theorem 1.4. The Li-Yorke chaotic eigen set of the positive integer multiple of backward shift operator on $\ell^{2}(\mathbb{N})$ :

*Corresponding Author: B. Sanooj: Department of Mathematics, College of Engineering Trivandrum, Thiruvananthapuram, APJ Abdul Kalam Technological University, India, E-mail: sanoojb.123@gmail.com

P.B. Vinodkumar: Centre for Topology and Applications, Department of Mathematics, Rajagiri School of Engineering and Technology, Kochi, APJ Abdul Kalam Technological University, India, E-mail: vinod_kumar@rajagiritech.edu.in 


$$
T\left(x_{1}, x_{2}, \cdots\right)=\left(x_{2}, x_{3}, \cdots\right)
$$

is $L Y(n T)=(n+1) \mathbb{D}$ for any $n>1, n \in \mathbb{N}$.

\section{Proof}

By definition, we have $L Y(n T)=\{\lambda \in \mathbb{C} / n T-\lambda I$ is Li-Yorke chaotic $\}$.

Now, for any $\lambda \in(n+1) \mathbb{D} \backslash\{0\}$, we can prove that $(-\lambda+n \mathbb{D}) \cap \mathbb{T} \neq \phi$.

For this, let us consider $w=\frac{1}{n}\left(\lambda-\frac{\lambda}{|\lambda|}\right)$.

Then

$$
\begin{aligned}
|w|^{2} & =w \bar{w} \\
& =\frac{1}{n}\left(\lambda-\frac{\lambda}{|\lambda|}\right) \frac{1}{n}\left(\bar{\lambda}-\frac{\bar{\lambda}}{|\bar{\lambda}|}\right) \\
& =\frac{1}{n^{2}}\left(\lambda \bar{\lambda}-\frac{\lambda \bar{\lambda}}{|\bar{\lambda}|}-\frac{\lambda \bar{\lambda}}{|\lambda|}+\frac{\lambda \bar{\lambda}}{|\lambda||\bar{\lambda}|}\right) \\
& =\frac{1}{n^{2}}\left(|\lambda|^{2}-2|\lambda|+1\right) \\
& =\frac{1}{n^{2}}(|\lambda|-1)^{2}
\end{aligned}
$$

Since $\lambda \in(n+1) \mathbb{D} \backslash\{0\}$, then we have $0<|\lambda|<n+1$. Hence $(|\lambda|-1)^{2}<n^{2}$. Thus $|w|^{2}<1$ which implies that $|w|<1$. Therefore, $w \in \mathbb{D}$ and hence $-\lambda+n w \in-\lambda+n \mathbb{D}$.

But

$$
\begin{aligned}
-\lambda+n w & =-\lambda+\lambda-\frac{\lambda}{|\lambda|} \\
& =-\frac{\lambda}{|\lambda|}
\end{aligned}
$$

Therefore, $|-\lambda+n w|=\left|\frac{-\lambda}{|\lambda|}\right|=1$ which implies that $-\lambda+n w \in \mathbb{T}$.

Thus we see that for any $\lambda \in(n+1) \mathbb{D} \backslash\{0\},-\lambda+n w$ belongs to both $-\lambda+n \mathbb{D}$ and $\mathbb{T}$ which shows that $(-\lambda+n \mathbb{D}) \cap \mathbb{T} \neq \phi$.

Now, we have $n T-\lambda I \in B_{n}(-\lambda+n \mathbb{D})$ and $(-\lambda+n \mathbb{D}) \cap \mathbb{T} \neq \phi$. By lemma 1.3, $n T-\lambda I$ is Li-Yorke chaotic. For the case when $\lambda=0, n T$ is Li-Yorke chaotic (see $[9,13])$. Thus we have shown that $n T-\lambda I$ is Li-Yorke chaotic for every $\lambda$ in the disk $(n+1) \mathbb{D}$.

Also, for any $\lambda \notin(n+1) \mathbb{D}$,

$$
\begin{aligned}
\|n T-\lambda I\| & \geq\|\lambda\|-n\|T\| \\
& \geq n+1-n \\
& \geq 1
\end{aligned}
$$

which shows that $n T-\lambda I$ is not Li-Yorke chaotic.

Thus we have shown that for any $\lambda \in(n+1) \mathbb{D}, n T-\lambda I$ is Li-Yorke chaotic and for any $\lambda \notin(n+1) \mathbb{D}, n T-\lambda I$ is not Li-Yorke chaotic, which proves that $L Y(n T)=(n+1) \mathbb{D}$.

Corollary:- $\bigcup_{n \in \mathbb{N}} L Y(n T)=\mathbb{C}$.

Acknowledgements: The authors would like to thank APJ Abdul Kalam Technological University, College of Engineering Trivandrum, Thiruvananthapuram and Rajagiri School of Engineering and Technology, Kochi for providing the facilities for the research work. 


\section{References}

[1] F. Bayart, E. Matheron, Dynamics of Linear Operators (1st ed.) (2009), Cambridge Tracts in Mathematics, 175, Cambridge University Press, Cambridge.

[2] T. Bermudez, A. Bonilla, F. Martnez Gimenez, A. Peris, Li-Yorke and distributionally chaotic operators, J. Math. Anal. Appl. (2011), 373, 83-93

[3] N.C Bernardes Jr, A. Bonilla, V. Muller, A. Peris, Li-yorke chaos in linear dynamics, Ergodic Theory Dynamic. Systems. (2014), 35, 1-23

[4] Bingzhe Hou, Lvlin Luo, Li-Yorke chaos translation set for linear operators, Arch. Math. @ 2018 Springer International Publishing AG, https://doi.org/10.1007/s00013-018-1176-y

[5] Bingzhe Hou, Puyu Cui, Yang Cao, Chaos for Cowen-Douglas Operators, Proc. Amer. Math. Soc. (2010), 138, 929-936

[6] Bingzhe Hou, Geng Tian, Luoyi Shi, Some Dynamical Properties For Linear Properties, Illinois J. Math. (2009), 53, 857-864

[7] K.C. Chan, The density of hypercyclic operators on a Hilbert space, J. Operator Theory. (2001), 47, 131-143

[8] R. L. Devaney, An introduction to Chaotic Dynamical Systems (2nd ed.) (1989), Addison-Wesley, Redwood City, CA.

[9] Emma D’Aniello, On Topological Chaos, Real Analysis Exchange.(2012), Summer Symposium XXXVI, 47-56

[10] X.C. Fu, J. Duan, Infinite-dimensional linear dynamical systems with chaoticity, J. Non-linear Sci. (1999), 9(2), 197-211

[11] Gabriel T. Prajitura, Irregular vectors of Hilbert space Operators, J. Math. Anal. Appl. (2009), 354, 689-697

[12] K. G. Grosse Erdmann, Recent developments in hypercyclicity, RACSAM Rev. R. Acad. Cienc. Exactas Fis. Nat. Ser. A Mat. (2003), 97, 273-286

[13] K. G. Grosse Erdmann, A. P. Manguillot, Linear Chaos (2011), Springer Universitext.

[14] T. Li, J. A. Yorke, Period three implies chaos, Amer. Math. Monthly. (1975), 82, 985-992

[15] X. Wu, P. Zhu, Li-Yorke chaos of backward shift operators on Kothe sequence spaces, Topology Appl. (2013), 160, 924-929 